\title{
Improved chemical-genetic fluorescent markers for live cell microscopy
}

\author{
Alison G. Tebo, Frederico M. Pimenta, Yu Zhang \& Arnaud Gautier* \\ PASTEUR, Département de Chimie, École Normale Supérieure, PSL University, Sorbonne Université, CNRS, 75005 Pa- \\ ris, France.
}

Supporting Information Placeholder

\begin{abstract}
Inducible chemical-genetic fluorescent markers are promising tools for live cell imaging requiring high spatiotemporal resolution and low background fluorescence. The Fluorescence-Activating and absorption Shifting Tag (FAST) was recently developed to form fluorescent molecular complexes with a family of small, synthetic fluorogenic chromophores (so-called fluorogens). Here, we use rational design to modify the binding pocket of the protein and screen for improved fluorescence performances with four different fluorogens. The introduction of a single mutation results in improvements in both quantum yield and dissociation constant with nearly all fluorogens tested. Our improved FAST (iFAST) allowed the generation of a tandem td-iFAST that forms green and red fluorescent reporters 1.6-fold and 2-fold brighter than EGFP and mCherry, respectively, while having comparable size.
\end{abstract}

The ubiquitous implementation of fluorescence microscopy to address biological questions has led to the development of a series of markers based on small molecules, proteins, and hybrid techniques. The discovery of the green fluorescent protein (GFP) and the subsequent expansion of the color palette of fluorescent proteins have revolutionized biological imaging due to being genetically encoded and their absolute labeling specificity. ${ }^{1}$ In parallel, a suite of small molecule probes with excellent brightness and photostability complements the toolbox of fluorescent markers. ${ }^{2}$ However, both approaches suffer from drawbacks - fluorescent proteins are large and have slow maturation times, while small molecule probes suffer from off-target labeling. Thus, hybrid techniques have sought to combine the advantages of the two approaches for live cell imaging, while circumventing their respective drawbacks. ${ }^{3}$

A particularly promising hybrid approach is the use of inducible chemical-genetic fluorescent markers, which are comprised of a small protein tag that interacts specifically with a small molecule ligand, termed fluorogen, whose fluorescence is increased upon interaction with its protein tag. ${ }^{4-6}$ A recently developed system named the Fluorescence-Activating and absorption Shifting Tag $(\text { FAST })^{7}$ is comprised of a small, monomeric, $14 \mathrm{kDa}$ protein that was engineered from the photoactive yellow protein (PYP) to specifically and reversibly bind a series of hydroxybenzylidene rhodanine (HBR) derivatives (Figure 1A,B). Upon interaction with FAST, the fluorogen is locked into an emissive conformation. The resulting fluorescent complex provides many of the advantages of traditional autofluorescent proteins for live cell imaging while circumventing slow maturation times and oxygen dependence. The bipartite nature of this type of system allows one to optimize the fluorogen and the protein tag independently to improve or fine-tune the properties of the system. Previously, optimization of the fluorogen generated a suite of brighter and redder fluorescent markers, while still retaining the same protein tag. ${ }^{8}$ To further improve the brightness, we optimized the protein tag through rational design of the binding pocket. The resulting variants were found to have increased quantum yields, particularly with the recently developed fluorogens HBR3OM and HBR-3,5DOM. ${ }^{8}$ We compared our rationally designed variants to new FAST variants that were recently identified through a new image-based, in-cell library screen to be brighter than FAST, but were not characterized as purified proteins. ${ }^{9}$ Finally, we present tandem constructs of FAST variants as an effective strategy to increase the overall brightness of the system.

The parent protein of FAST, PYP, is a bacterial photoreceptor with a covalently attached 4-hydroxycinnamoyl (HC) chromophore, which participates in hydrogen bonding with Y42 and E46 (Figure 1C). ${ }^{10-12}$ FAST was engineered through directed evolution of PYP by saturation mutagenesis of the loops near the chromophore binding pocket, resulting in replacement of the loop 94-101 in conjunction with the mutation of $\mathrm{C} 69$, which tethers $\mathrm{HC}$, to glycine. However, the rest of the chromophore pocket of PYP remains intact, which can inform our understanding of how HBR derivatives bind to FAST. The observed 
A
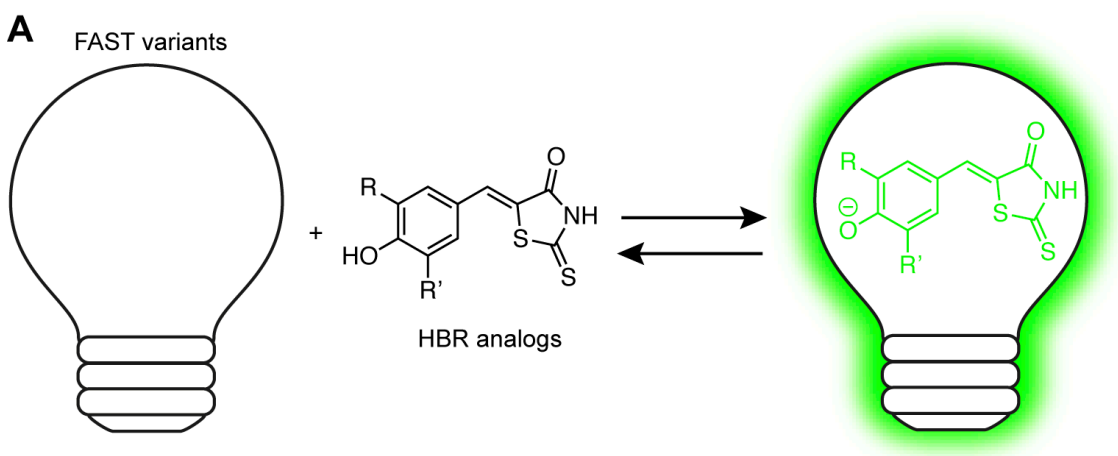

C

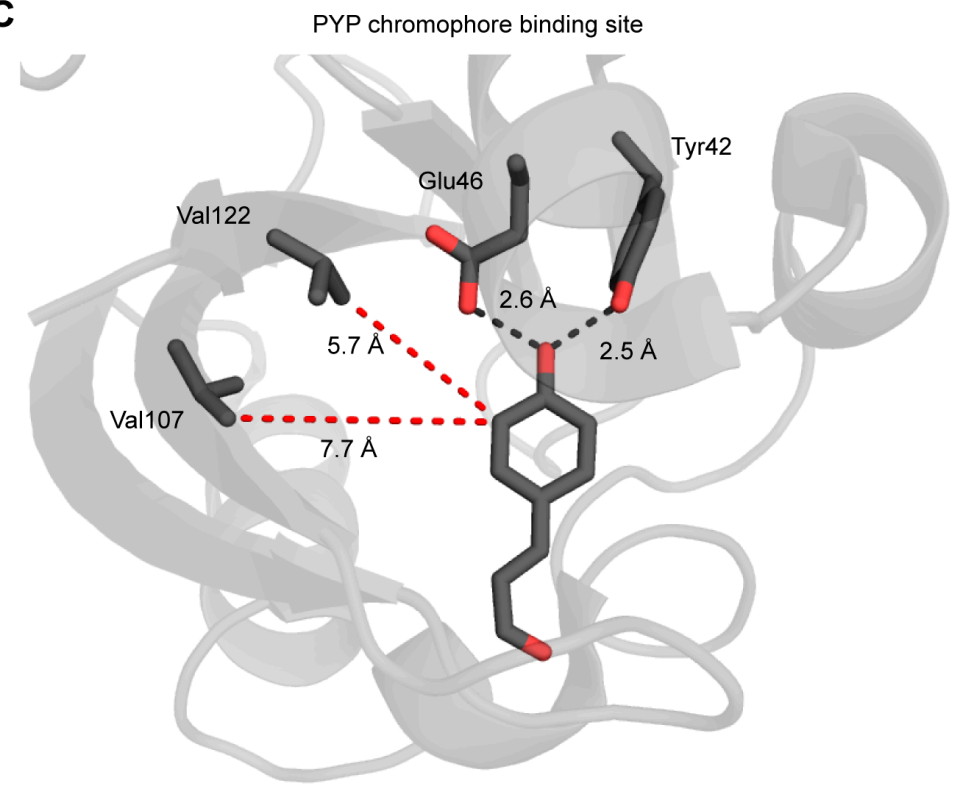

D
B<smiles>Cc1ccc(/C=C2\SC(=S)NC2=O)c(O)c1</smiles><smiles>COc1cc(/C=C2\SC(=S)NC2=O)cc(OC)c1O</smiles>

Hypothetical binding site in iFAST

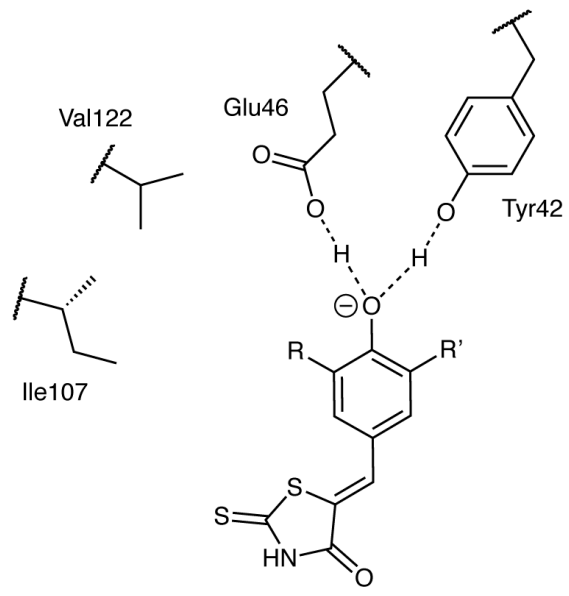

Figure 1. FAST principle and improvement. (A) FAST variants bind and activate the fluorescence of HBR analogs. (B) The structures of the HBR analogs used in this study. (C) Detailed view of the chromophore binding pocket in PYP including the relative positions of V107 and V122 (PDB: 1nwz). (D) Proposed fluorogen binding pocket in iFAST.

characteristics of FAST, namely the absorption red-shift and fluorescence activation upon fluorogen binding are due to the binding mode of the fluorogen to the protein. The absorption red-shift is in agreement with the fluorogen being bound in its deprotonated form and stabilized by the same amino acids that interact with the chromophore of PYP, Y42 and E46. Furthermore, binding restricts the conformational freedom of the chromophore, leading to fluorescence activation. In seeking to improve the fluorescence quantum yield of FAST, we sought to further increase the conformational locking of the chromophore in the binding pocket. We identified the residues V107 and V122 as being potential targets for rational design because they are about 8 and $6 \AA$ from the benzyl ring of 4-hydroxycinnamic acid in the PYP crystal structure (PDB: 1NWZ). ${ }^{13}$ Since the most successful fluorogens yet developed for FAST include modifications to the benzyl moiety to increase bulk, ${ }^{8}$ we hypothesized that further introduction of bulky residues at these positions in the binding pocket of the protein would further improve the physicochemical properties of the complex (Figure 1D).
The proteins FAST ${ }^{\mathrm{V} 107 \mathrm{I}}, \mathrm{FAST}^{\mathrm{V} 122 \mathrm{I}}$, and double mutant $\mathrm{FAST}^{\mathrm{V} 107 \mathrm{I}, \mathrm{V} 122 \mathrm{I}}$ were generated by site-directed mutagenesis and expressed in E. coli. The mutants reported by Emanuel et al, ${ }^{9}$ mutant 1 , mutant 2 , and mutant3, were also expressed and purified. The thermodynamic dissociation constant $K_{\mathrm{D}}$, fluorescence quantum yield $(\Phi)$, and molar absorption coefficient were measured for the 24 pairs made of the six proteins with the four fluorogens: HMBR, HBR-3,5DM, HBR-3OM, and HBR-3,5DOM (Table 1, Figures 2 and S1). The mutation V107I was observed to slightly increase the affinity of the protein for the fluorogens. Furthermore, for certain fluorogens, particularly HBR-3OM and HBR-3,5DOM, which have bulky methoxy substituents on the benzyl ring, the quantum yield was dramatically increased, while the quantum yield for HBR-3,5DM exhibited a more modest increase in quantum yield. On the other hand, the mutation V122I decreased the binding affinity for the fluorogens by a factor of 3 to 8 , depending on the fluorogen. This loss of affinity might result from V122I being closer to the fluorogen binding site relative to V107I. Nonetheless, this mutation also increased the quantum yield of several of the 
Table 1. Physico-chemical properties of mutant:fluorogen complexes in PBS pH 7.4. Abbreviations are as follows: $\lambda_{\text {abs, }}$ wavelength of maximal absorption; $\lambda_{\mathrm{em}}$, wavelength of maximal emission; $\varepsilon$, molar absorption coefficient at $\lambda_{\text {abs }}$ (standard error is typically $10 \%$ ); $\Phi$, fluorescence quantum yield; $K_{\mathrm{D}}$ thermodynamic dissociation constant.

\begin{tabular}{|c|c|c|c|c|c|c|}
\hline Mutant & Fluorogen & $\lambda_{\text {abs }}(\mathrm{nm})$ & $\lambda_{\mathrm{em}}(\mathrm{nm})$ & $\varepsilon\left(\mathrm{mM}^{-1} \mathrm{~cm}^{-1}\right)$ & $\Phi$ & $\begin{array}{l}K_{\mathrm{D}} \\
(\mu \mathrm{M})\end{array}$ \\
\hline \multirow[t]{4}{*}{ FAST } & HMBR & 481 & 540 & 45 & $0.23 \pm 0.03$ & 0.13 \\
\hline & HBR-3,5DM & 499 & 562 & 48 & $0.49 \pm 0.05$ & 0.08 \\
\hline & HBR-3OM & 494 & 561 & 40 & $0.36 \pm 0.04$ & 0.31 \\
\hline & HBR-3,5-DOM & 518 & 600 & 39 & $0.31 \pm 0.03$ & 0.97 \\
\hline \multirow[t]{4}{*}{ iFAST } & HMBR & 480 & 541 & 41 & $0.22 \pm 0.03$ & 0.07 \\
\hline & HBR-3,5DM & 499 & 558 & 46 & $0.57 \pm 0.08$ & 0.06 \\
\hline & HBR-3OM & 495 & 560 & 39 & $0.49 \pm 0.07$ & 0.2 \\
\hline & HBR-3,5-DOM & 516 & 600 & 38 & $0.40 \pm 0.04$ & 0.41 \\
\hline \multirow[t]{4}{*}{ FAST $^{\mathrm{V} 122 \mathrm{I}}$} & HMBR & 480 & 542 & 41 & $0.35 \pm 0.05$ & 0.35 \\
\hline & HBR-3,5DM & 499 & 559 & 43 & $0.52 \pm 0.07$ & 0.57 \\
\hline & HBR-3OM & 495 & 560 & 38 & $0.47 \pm 0.07$ & 1.4 \\
\hline & HBR-3,5-DOM & 516 & 596 & $\sim 30$ & $0.32 \pm 0.03$ & 8.3 \\
\hline \multirow[t]{4}{*}{ FAST $^{\mathrm{V} 107 \mathrm{I}, \mathrm{V} 122 \mathrm{I}}$} & HMBR & 480 & 541 & 35 & $0.24 \pm 0.03$ & 0.3 \\
\hline & HBR-3,5DM & 499 & 558 & 50 & $0.48 \pm 0.07$ & 0.29 \\
\hline & HBR-3OM & 495 & 560 & 33 & $0.49 \pm 0.07$ & 2.0 \\
\hline & HBR-3,5-DOM & 516 & 598 & $\sim 30$ & $0.33 \pm 0.04$ & 6.4 \\
\hline \multirow[t]{4}{*}{ mutant1 } & HMBR & 486 & 545 & 56 & $0.23 \pm 0.03$ & 0.024 \\
\hline & HBR-3,5DM & 507 & 565 & 48 & $0.25 \pm 0.03$ & 0.58 \\
\hline & HBR-3OM & 501 & 555 & 51 & $0.26 \pm 0.03$ & 0.17 \\
\hline & HBR-3,5-DOM & 515 & 595 & 36 & $0.26 \pm 0.03$ & 2.0 \\
\hline \multirow[t]{4}{*}{ mutant2 } & HMBR & 486 & 544 & 59 & $0.23 \pm 0.03$ & 0.024 \\
\hline & HBR-3,5DM & 504 & 565 & 47 & $0.34 \pm 0.04$ & 0.49 \\
\hline & HBR-3OM & 501 & 555 & 48 & $0.26 \pm 0.03$ & 0.2 \\
\hline & HBR-3,5-DOM & 516 & 595 & 41 & $0.27 \pm 0.03$ & 2.5 \\
\hline \multirow[t]{4}{*}{ mutant3 } & HMBR & 488 & 547 & 39 & $0.27 \pm 0.04$ & 0.012 \\
\hline & HBR-3,5DM & 504 & 565 & 47 & $0.34 \pm 0.04$ & 0.21 \\
\hline & HBR-3OM & 502 & 555 & 50 & $0.24 \pm 0.03$ & 0.2 \\
\hline & HBR-3,5-DOM & 515 & 596 & 38 & $0.25 \pm 0.03$ & 3.0 \\
\hline
\end{tabular}

complexes. The effect of the two mutations was not additive, as seen from the characterization of the double mutant FAST ${ }^{\mathrm{V} 107 \mathrm{I}, \mathrm{V} 122 \mathrm{I}}$, which displayed lower binding affinities as might be expected from the introduction of V122I and similar increases in quantum yield as previously observed in both single mutants. The overall brightest complex is FAST $^{\mathrm{V} 107 \mathrm{I}}$ :HBR-3,5DM, while the largest improvement in quantum yield was achieved with FAST $^{\mathrm{V} 1071}$ :HBR-3OM, thus we termed this protein iFAST for improved-FAST. Interestingly, the mutant1-3 that were reported previously as brighter than FAST:HMBR by in-cell measurement in E. coli showed no significant improvement in fluorescence quantum yield relative to FAST, although the binding affinity for HMBR was increased by an order of magnitude. When tested with the three other fluorogens, mutants 1-3 showed poorer performance, exhibiting lower quantum yields overall. The reported improvement in mutant 1-3 brightness with HMBR in $E$. coli cells is probably due to the increased binding affinity favoring reporter formation in bacteria.

Next, we hypothesized that dimerizing FAST could generate significantly brighter markers for imaging through increase of the apparent absorption coefficient, as 

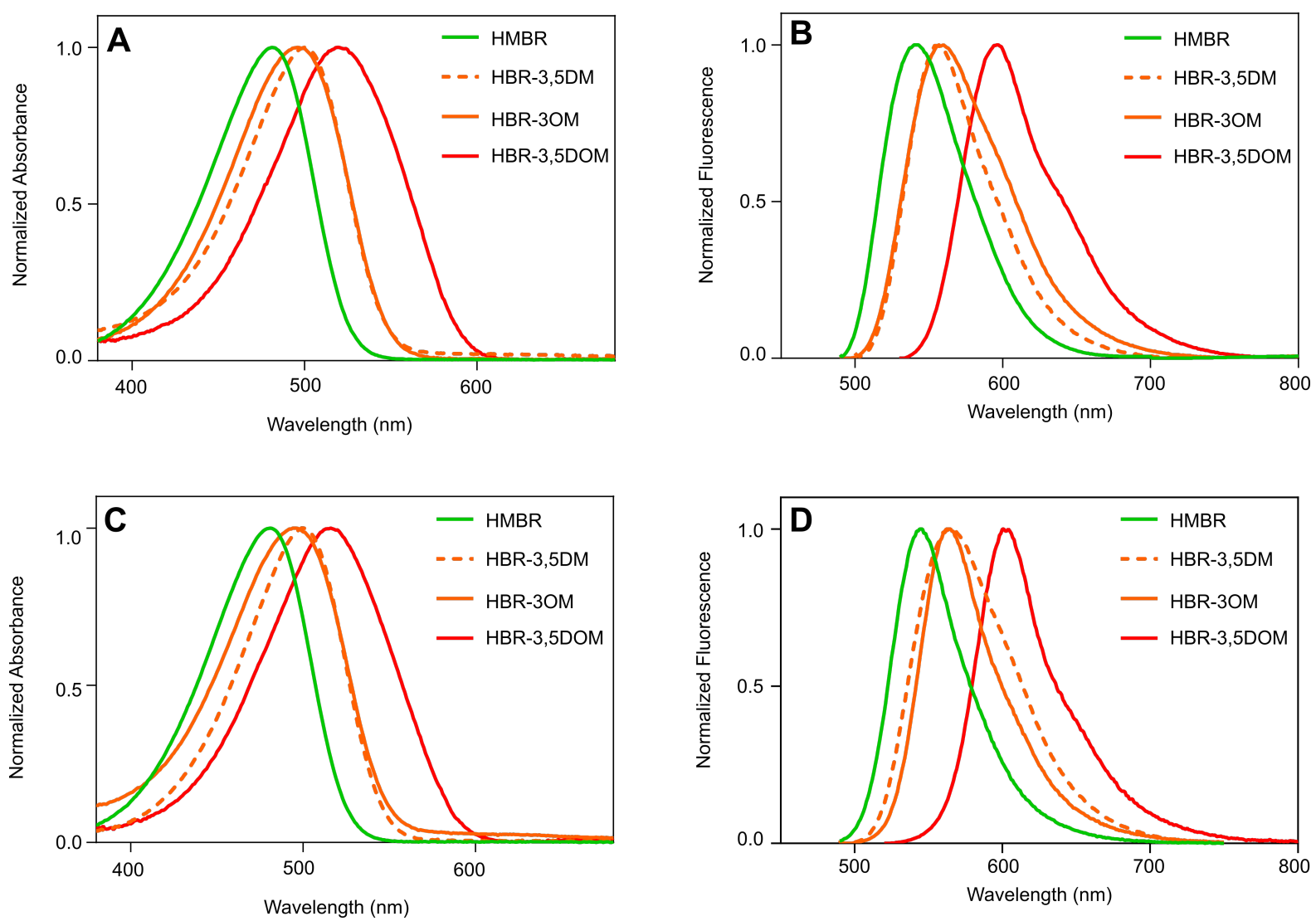

Figure 2. Spectral characteristics of FAST and iFAST. Absorption spectra of FAST (A) and iFAST (C) and fluorescence spectra of FAST (B) and iFAST (D) with HMBR, HBR-3,5DM, HBR-3OM, and HBR-3,5DOM. Fluorogen concentration was $3 \mu \mathrm{M}$ and FAST mutant concentration was $40 \mu \mathrm{M}$. Spectra were recorded in $\mathrm{pH} 7.4 \mathrm{PBS}$ at $25^{\circ} \mathrm{C}$.

has previously been done with some autofluorescent proteins such as tdTomato. ${ }^{14} \mathrm{~A}$ tandem of FAST (td-FAST) results in a probe of about $28 \mathrm{kDa}$, which is about the same size as fluorescent proteins, with a brightness 1.4fold higher than EGFP ${ }^{15}$ when used with HBR-3,5DM. Furthermore, to capitalize on the mutations that improve the quantum yield of FAST, we generated a tandem of iFAST (td-iFAST) as well, which provides an additional improvement in overall brightness depending on the fluorogen chosen. The brightness of td-FAST and td-iFAST along with FAST and iFAST was tested in live cell confocal microscopy with HBR-3OM, which exhibits the largest change in quantum yield between FAST and iFAST, to evaluate their relative brightness in the context of cell imaging (Figure 3). We observed that tandems of FAST are expressed homogenously in the cell, confirming that neither the size nor the construction of the tandem impedes diffusion into the nucleus (Figure 3A). Relative to FAST, iFAST was found to be 1.7-fold brighter, which is slightly higher than predicted based on the brightness measured in vitro (Figure 3B,C and Figures S2-S3). Furthermore, td-FAST and td-iFAST were found to be 2.8fold and 3.8-fold brighter than FAST, respectively (Figure $3 \mathrm{~B}, \mathrm{C}$ and Figures $\mathrm{S} 2-\mathrm{S} 3$ ).

Next we examined the photobleaching of FAST, iFAST, FAST $^{\mathrm{V} 122 \mathrm{I}}$, and mutant 1 with HMBR in living cells. It has previously been reported that FAST exhibits a biphasic photobleaching behavior composed of a rapid phase and a slow phase. ${ }^{9,16}$ Long acquisitions by confocal microscopy allowed us to compare the photobleaching behavior of the four proteins with $10 \mu \mathrm{M}$ HMBR (Figure S4A). In the conditions used, iFAST, FAST ${ }^{\mathrm{V} 122 \mathrm{I}}$, and mutant1 show a reduction of the amplitude of the rapid bleaching phase compared to FAST. After this rapid initial fluorescence decrease, FAST, iFAST, FAST ${ }^{\text {V122I }}$ showed almost steady fluorescence, while mutant1 seemed to be slightly more prone to photodamage (Figure S4A). Mutant1 contains a cysteine residue, which is prone to photooxidation, in its sequence that is absent in the three other variants, which might explain the observed difference of behavior. The photobleaching of FAST and iFAST were also examined in the presence of $10 \mu \mathrm{M}$ HBR-3,5DM, HBR-3OM, and HBR-3,5DOM (Figure S4B-D). FAST and iFAST showed similar photobleaching curves for all three fluorogens. The reporters with HBR-3,5DM and HBR-3OM, while being brighter, appear to be slightly more sensitive to photobleaching than with HMBR, particularly in the case of HBR-3OM. Imaging with $10 \mu \mathrm{M}$ HBR-3,5DOM revealed that both FAST and iFAST are as photostable as mCherry.

One major advantage of fluorogenic and hybrid systems comprised of a small molecule and a protein tag is that further development of the system can be done by 

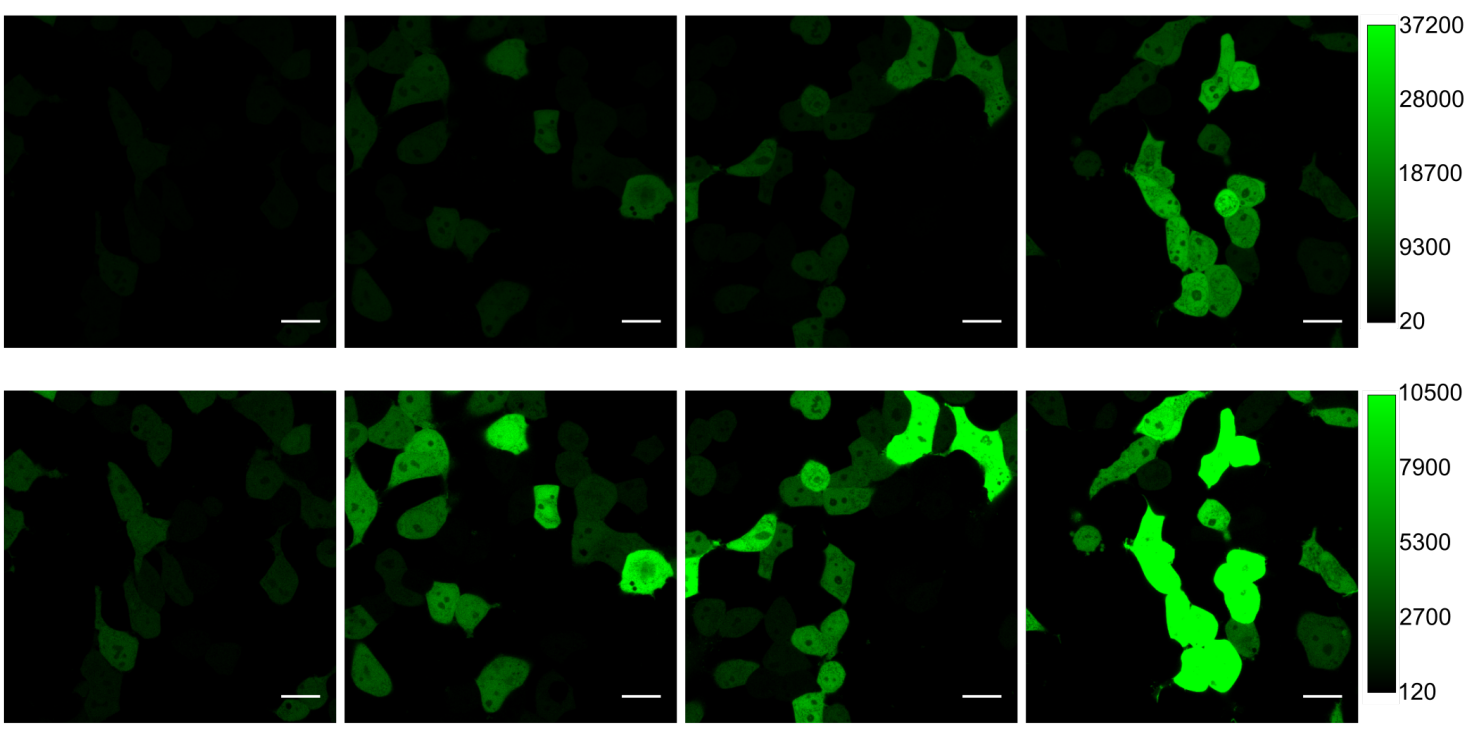

B

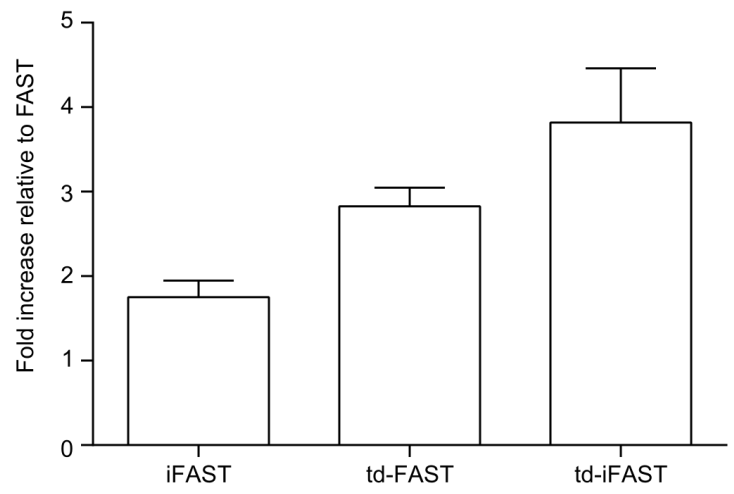

C

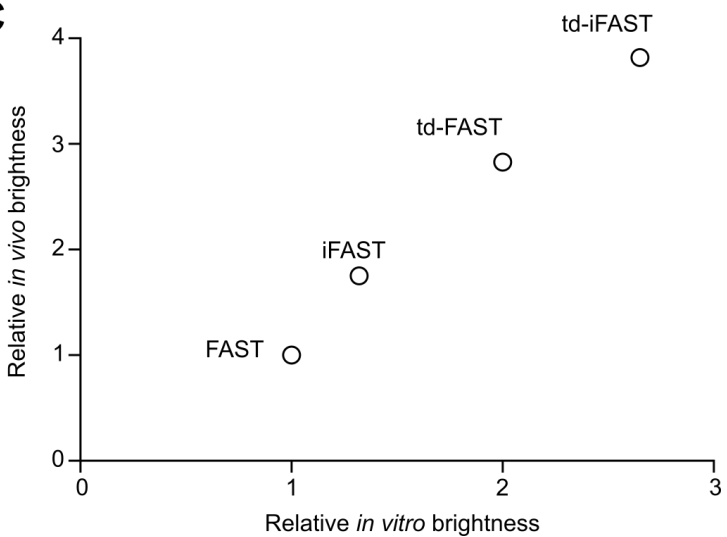

Figure 3. Relative in-cell brightness of FAST, iFAST, td-FAST, and td-iFAST. (A) Representative confocal micrographs of HEK 293T cells expressing FAST, iFAST, td-FAST, and td-iFAST. The images were all acquired at 16-bit with the same settings, each pixel defined by 65,536 grey levels. Given the difference of brightness between FAST, iFAST, td-FAST and tdiFAST, the intensity scale used for the images of tdFAST and td-iFAST did not allow the proper visualization of FAST and iFAST. Therefore we provided one panel (top) that is optimized for visualizing td-FAST and a second (bottom) that is optimized for visualizing FAST. Intensity (grey level) scales are given on the right of each panel. Cells were labeled with $20 \mu \mathrm{M} \mathrm{HBR}-$ 3OM. Scale bars are $20 \mu \mathrm{m}$. (B) Relative brightness in cells (mean \pm sem of 4 experiments, each with an average of 80-90 cells quantified). Fluorescence levels were normalized by the protein expression level determined by quantitative western blotting as shown on Figure S2. Figure S3 presents the individual measurements. (C) Comparison of the brightness of FAST, iFAST, td-FAST and td-iFAST labeled with HBR-3OM in mammalian cells (in vivo brightness) and solution (in vitro brightness).

optimizing either the protein or the small molecule. Here, we have presented work to improve the fluorescence performance of the FAST system by manipulation of the protein tag by rational design to introduce a single mutation that increases steric hindrance around the chromophore. The use of rational design was complementary to the directed evolution approach used originally for developing FAST, as it has facilitated the finetuning of properties difficult to further improve by high-throughput approaches. Not only has rational design provided us with improved tags but also supports our hypothesis that HBR derivatives bind to FAST in a similar fashion as 4-hy- droxycinnamic acid to PYP. We also showed that dimerizing FAST is an effective way to increase the overall brightness, particularly when the size of the tag is less of an issue. Optimized td-iFAST is 1.6-fold brighter than EGFP, while having comparable size. Since the color of FAST-based systems can be changed at-will, td-iFAST presents a particularly attractive alternative to red fluorescent proteins. In particular, td-iFAST with HBR-3,5DOM is 2-fold brighter than mCherry. These new variants of FAST exhibit quantum yields on par with fluorescent proteins while retaining the advantages of the FAST system - namely, no maturation time, on-demand imaging, small tag size, and the ability to change the color at-will. 
Furthermore, the introduction of these mutations demonstrates that FAST could be further modified in efforts to expand the FAST system, such as to further improve the fluorescence performance and physicochemical properties, but also to expand the color palette or engineer new fluorogen specificity.

\section{ASSOCIATED CONTENT}

Supporting Information. Supporting information contains Figures S1-S3 and Materials \& Methods. The Supporting Information is available free of charge on the ACS Publications website.

\section{AUTHOR INFORMATION}

\section{Corresponding Author}

*Arnaud Gautier arnaud.gautier@ens.fr

\section{Notes}

The authors declare the following competing financial interest: A.G. is co-founder and holds equity in Twinkle Bioscience, a company commercializing the FAST technology.

\section{ACKNOWLEDGMENT}

(This work has been supported by the European Research Council (ERC-2016-CoG-724705 FLUOSWITCH), the Agence National de la Recherche (ANR-14-CE09-000201), France Biolmaging (ANR-10-INBS-04), and the Equipex Morphoscope 2 (ANR-11-EQPX-0029).

\section{REFERENCES}

(1) Specht, E. A., Braselmann, E., and Palmer, A. E. (2017) A Critical and Comparative Review of Fluorescent Tools for Live-Cell Imaging. Annu. Rev. Physiol. 79, 93-117.

(2) Zheng, Q., and Lavis, L. D. (2017) ScienceDirect Development of photostable fluorophores for molecular imaging. Curr. Op. Chem. Biol. 39, 32-38.
(3) Dean, K. M., and Palmer, A. E. (2014) Advances in fluorescence labeling strategies for dynamic cellular imaging. Nat. Chem. Biol. 10, $512-523$.

(4) Bruchez, M. P. (2015) Dark dyes-bright complexes: fluorogenic protein labeling. Curr. Op. Chem. Biol. 27, 18-23.

(5) Jullien, L., and Gautier, A. (2015) Fluorogen-based reporters for fluorescence imaging: a review. Methods Appl. Fluoresc. 3, 04200713.

(6) Li, C., Tebo, A. G., and Gautier, A. (2017) Fluorogenic Labeling Strategies for Biological Imaging. IJMS 18, 1473-11.

(7) Plamont, M.-A., Billon-Denis, E., Maurin, S., Gauron, C., Pimenta, F. M., Specht, C. G., Shi, J., Querard, J., Pan, B., Rossignol, J., Morellet, N., Volovitch, M., Lescop, E., Chen, Y., Triller, A., Vriz, S., Le Saux, T., Jullien, L., and Gautier, A. (2016) Small fluorescenceactivating and absorption-shifting tag for tunable protein imaging in vivo. Proc. Natl. Acad. Sci. U. S. A 113, 497-502.

(8) Li, C., Plamont, M.-A., Sladitschek, H. L., Rodrigues, V., Aujard, I., Neveu, P., Le Saux, T., Jullien, L., and Gautier, A. (2017) Dynamic multicolor protein labeling in living cells. Chem. Sci. 8, 5598-5605.

(9) Emanuel, G., Moffitt, J. R., and Zhuang, X. (2017) Highthroughput, image-based screening of pooled genetic-variant libraries. Nat. Methods 14, 1159-1162.

(10) Baca, M., Borgstahl, G., Boissinot, M., Burke, P. M., Williams, D. W., Slater, K. A., and Getzoff, E. D. (1994) Complete chemical structure of photoactive yellow protein: novel thioester-linked 4-hydroxycinnamyl chromophore and photocycle chemistry. Biochemistry 33, 14369-14377.

(11) Borgstahl, G., Williams, D., and Getzoff, E. D. 1.4. Ang. structure of photoactive yellow protein, a cytosolic photoreceptor: Unusual fold, active site, and chromophore. Biochemistry 34, 6278-6287.

(12) Imamoto, Y., and Kataoka, M. (2007) Structure and Photoreaction of Photoactive Yellow Protein, a Structural Prototype of the PAS Domain Superfamily. Photochem. Photobiol. 83, 40-49.

(13) Getzoff, E. D., Gutwin, K. N., and Genick, U. K. (2003) Anticipatory active-site motions and chromophore distortion prime photoreceptor PYP for light activation. Nat. Struct. Biol. 10, 663-668.

(14) Shaner, N. C., Campbell, R. E., Steinbach, P. A., Giepmans, B. N. G., Palmer, A. E., and Tsien, R. Y. (2004) Improved monomeric red, orange and yellow fluorescent proteins derived from Discosoma sp. red fluorescent protein. Nat Biotechnol 22, 1567-1572.

(15) Day, R. N., and Davidson, M. W. (2009) The fluorescent protein palette: tools for cellular imaging. Chem Soc Rev 38, 2887-36.

(16) Pimenta, F. M., Chiappetta, G., Le Saux, T., Vinh, J. X. L., Jullien, L., and Gautier, A. (2017) Chromophore Renewal and FluorogenBinding Tags: A Match Made to Last. Scientific Reports 7, 12316. 


\title{
Supporting Information
}

Improved chemical-genetic fluorescent markers for live cell microscopy

\author{
Alison G. Tebo, Frederico M. Pimenta, Yu Zhang \& Arnaud Gautier* \\ PASTEUR, Département de Chimie, École Normale Supérieure, PSL \\ University, Sorbonne Université, CNRS, 75005 Paris, France. \\ * Correspondence should be addressed to: arnaud.gautier@ens.fr
}

\section{Content}

SI Figures: Fig. S1-S4

Materials \& Methods

SI References 

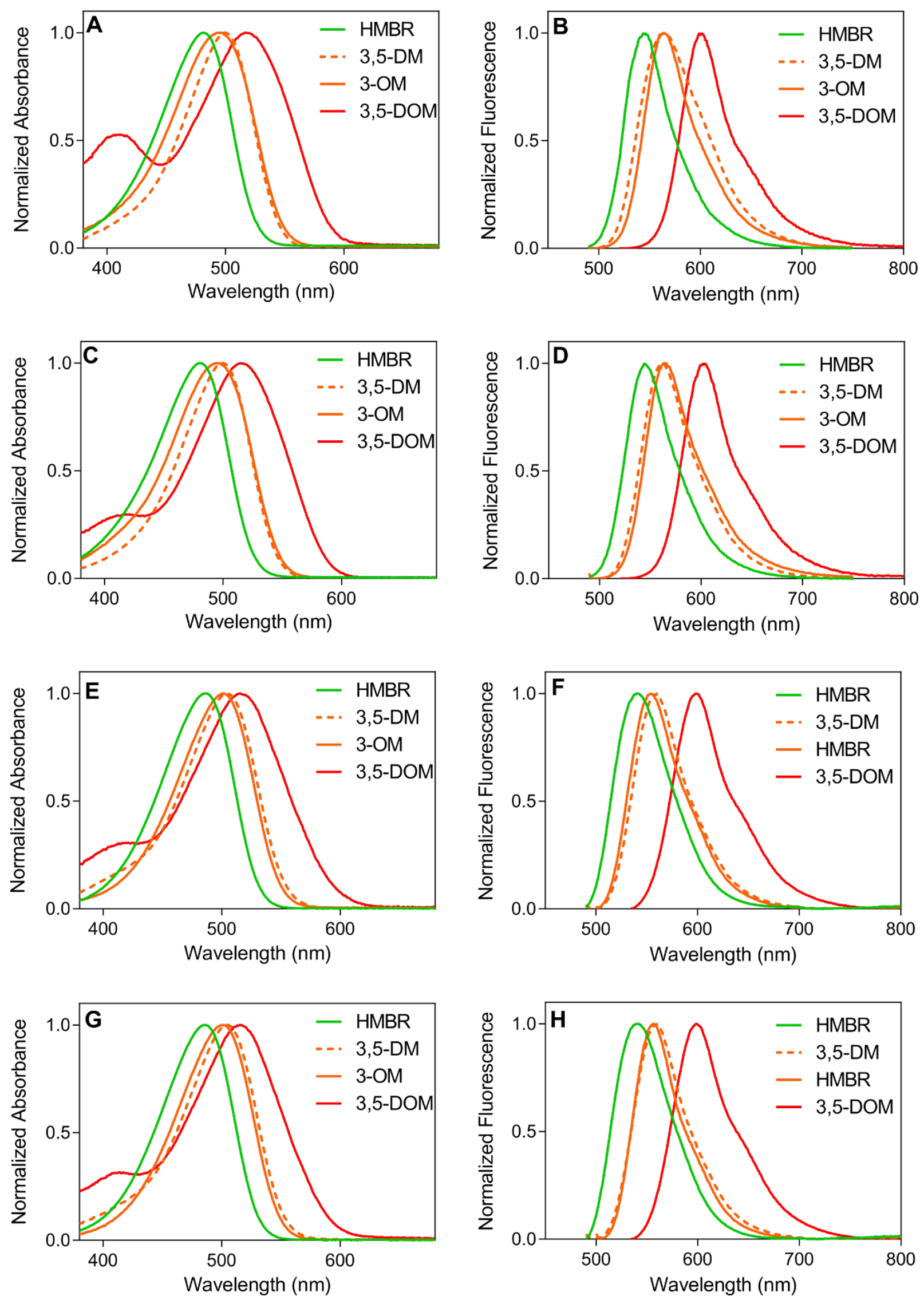

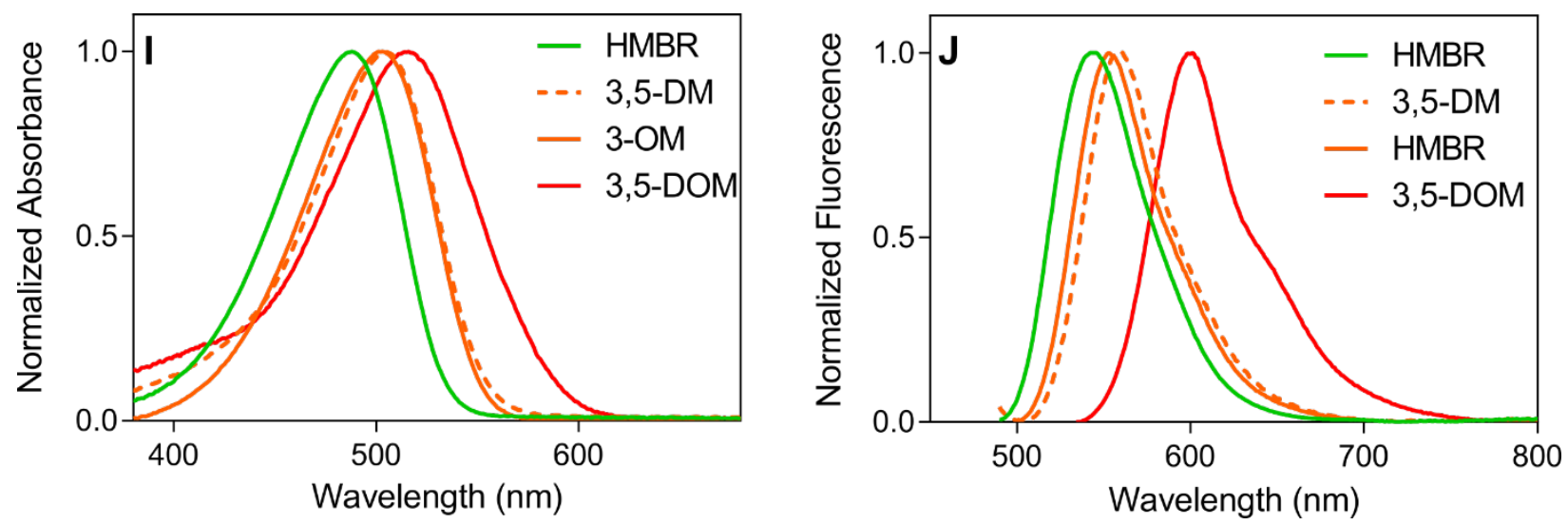

Figure S1. Absorbance and fluorescence spectra of FAST ${ }^{\mathrm{V} 122 !}$ (A, B), FAST ${ }^{\mathrm{V} 1071, \mathrm{~V} 1221}$ (C, $D)$, mutant1 $(E, F)$, mutant $2(G, H)$, mutant3 $(I, J)$. Fluorogen concentration was $3 \mu \mathrm{M}$ and FAST mutant concentration was $40 \mu \mathrm{M}$. Spectra were recorded in $\mathrm{pH} 7.4$ PBS at $25^{\circ} \mathrm{C}$. 
A

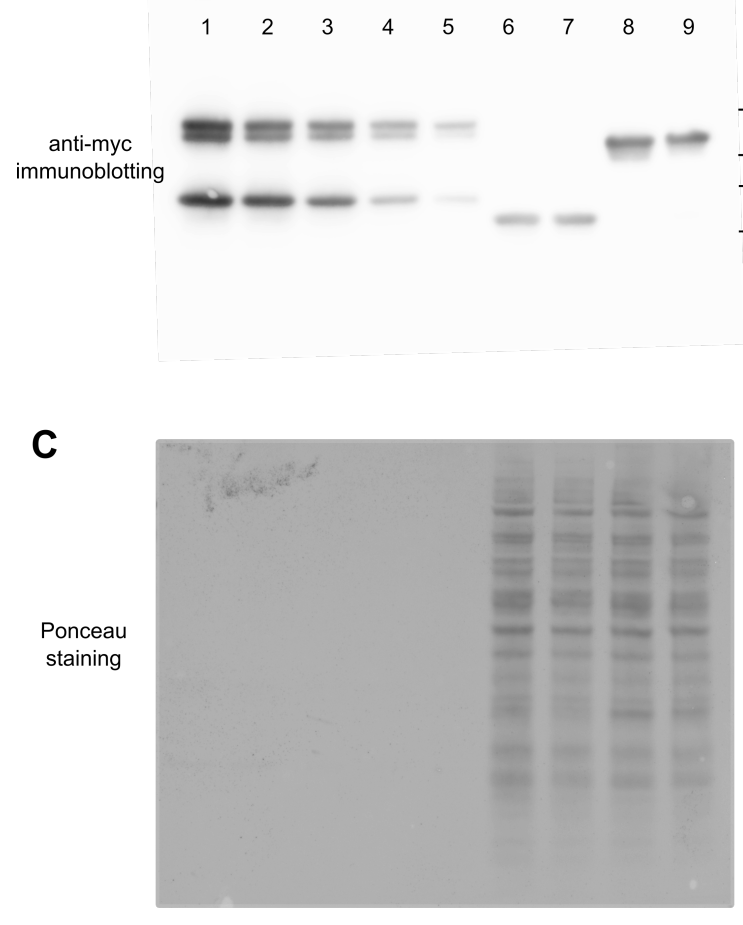

B

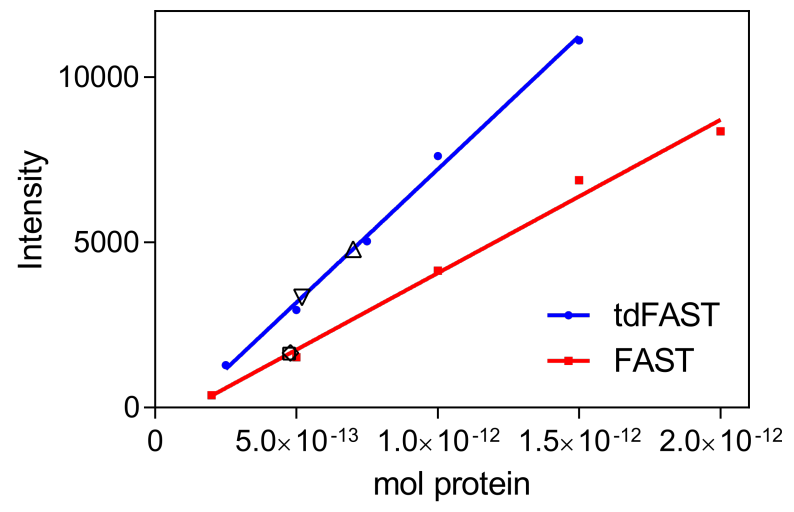

Figure S2. Quantification of protein concentration in microscopy samples. (A) Representative western. Lanes 1-5 are standards of Histag-tdFAST-myc and HistagFAST-myc. The following four lanes (6-9) are microscopy samples: FAST-myc, iFASTmyc, td-iFAST-myc, and td-FAST-myc. Immunoblotting was performed using an anti-myc antibody. (B) Standard curves of intensity of chemiluminescence versus mol of protein for lanes 1-6. The quantity of protein from microscopy samples was interpolated from the standard curves for td-FAST (inverted triangle), td-iFAST (triangle), FAST (square), and iFAST (diamond) (note that square and diamond are overlaid in this example). (C) Ponceau staining of membrane before incubation with anti-myc antibody. 


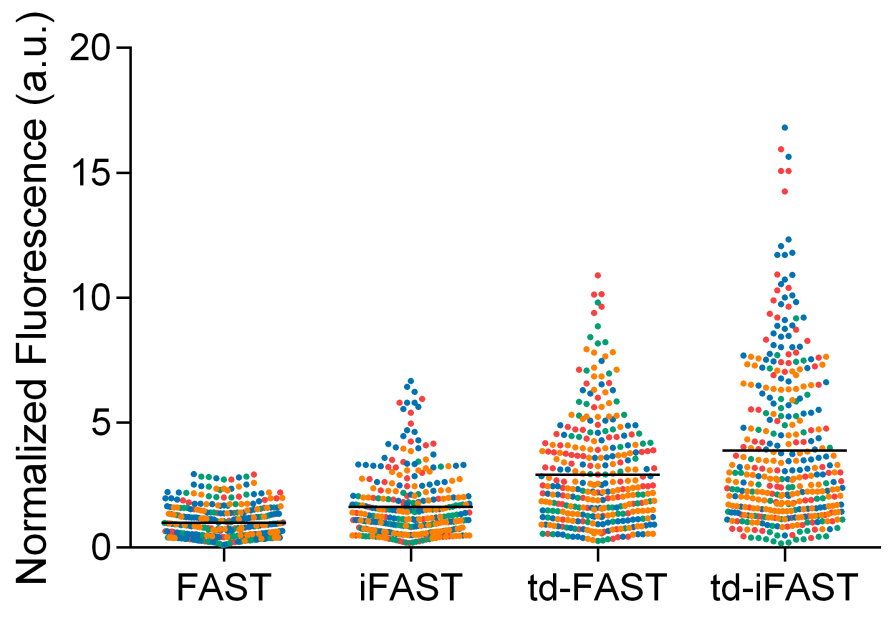

Figure S3. In-cell brightness of FAST, iFAST, td-FAST, and td-iFAST. Dot plot of all individual measurements of fluorescence intensity colored by experiment $(n=4$ different experiments). Mean fluorescence is indicated by black line. Values were normalized by protein expression level (see Figure S2) and mean fluorescence of FAST was set to 1 for comparison. 
A

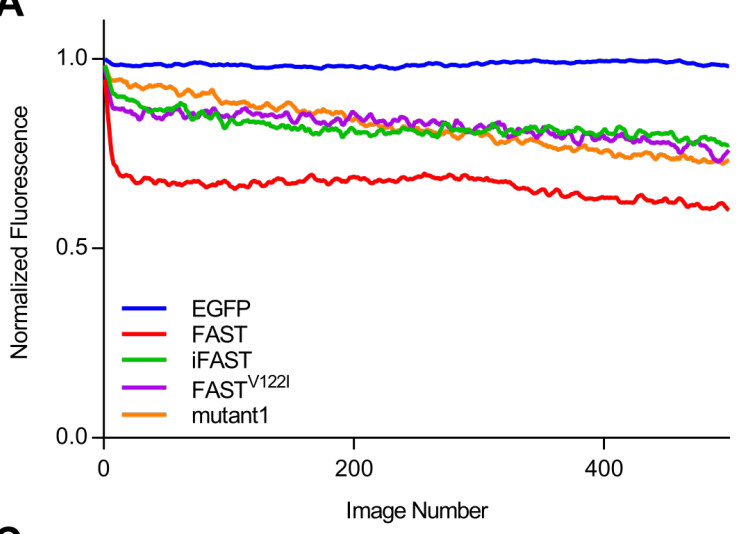

C

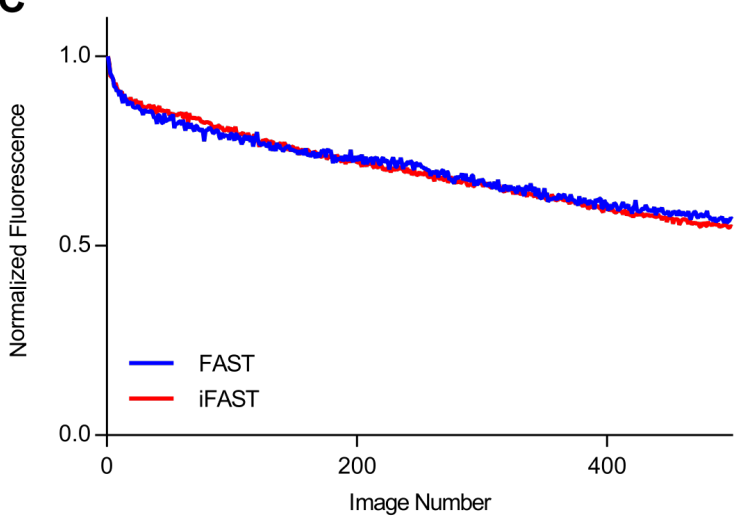

B

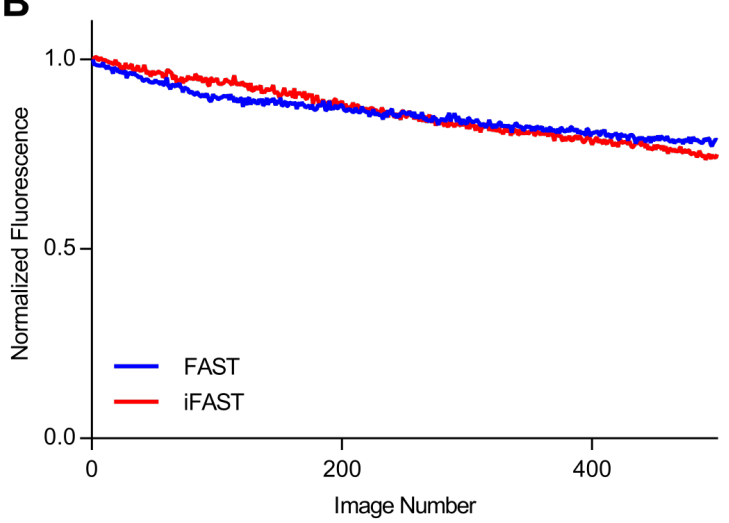

D

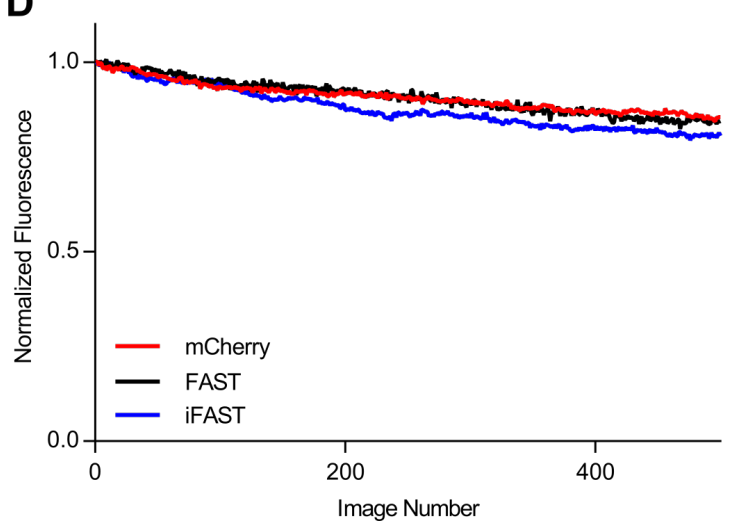

Figure S4. In-cell photobleaching of FAST and iFAST. (A) Comparison of photobleaching behavior between EGFP, FAST, iFAST, FAST ${ }^{\mathrm{V} 122 \mathrm{I}}$, and mutant1 in the presence of $10 \mu \mathrm{M} \mathrm{HMBR}$. (B) Comparison of photobleaching of FAST and iFAST in the presence of $10 \mu \mathrm{M}$ HBR-3,5DM. (C) Comparison of photobleaching of FAST and iFAST in the presence of $10 \mu \mathrm{M} \mathrm{HBR-3OM}$. (D) Comparison of photobleaching of mCherry, FAST and iFAST in the presence of $10 \mu \mathrm{M}$ HBR3,5DOM. (A-D) HEK 293T cells expressing the different FAST mutants were imaged using a scanning confocal microscope equipped with a $488 \mathrm{~nm}$ laser (with a power of $4.6 \mathrm{~kW} / \mathrm{cm}^{2}$ at the specimen plane) and a $541 \mathrm{~nm}$ laser (with a power of $2.7 \mathrm{~kW} / \mathrm{cm}^{2}$ at the specimen plane) at a frame rate of 1 image / $750 \mathrm{~ms}$ (pixel dwell time of $1.27 \mu \mathrm{s}$ ), $\mathrm{n}=3$ cells per curve. 


\section{MATERIALS AND METHODS}

General FAST is a variant of the photoactive yellow protein (PYP) containing the mutations C69G, Y94W, T95M, F96I, D97P, Y98T, Q99S, M100R, T101G. HMBR, HBR3,5DM, HBR-3OM, and HBR-3,5-DOM were prepared as previously described ${ }^{1,2}$.

Molecular Biology The point mutations were inserted in FAST via site-directed mutagenesis using complementary primers and Gibson assembly using the previously reported pAG87. The tandem construct was synthesized by Eurofins and subcloned into plasmids for bacterial expression using Nhe I and Xho I restriction sites. Mutations were subsequently introduced sequentially in the same manner as for FAST. The genes for mutants (1-3) were synthesized by Eurofins and subcloned into plasmids for bacterial expression by Gibson assembly. All proteins were then subcloned into plasmids for mammalian expression by Gibson assembly.

Protein expression and purification Expression vectors were transformed in Rosetta (DE3) pLysS E. coli (New England Biolabs). Cells were grown at $37^{\circ} \mathrm{C}$ in LB medium complemented with $50 \mu \mathrm{g} / \mathrm{ml}$ kanamycin and $34 \mu \mathrm{g} / \mathrm{ml}$ chloramphenicol to OD $_{600 \mathrm{~nm}}$ 0.6. Expression was induced for $4 \mathrm{~h}$ by adding isopropyl $\beta$-D-1-thiogalactopyranoside (IPTG) to a final concentration of $1 \mathrm{mM}$. Cells were harvested by centrifugation $(4,000 \times \mathrm{g}$ for 20 min at $4^{\circ} \mathrm{C}$ ) and frozen. The cell pellet was resuspended in lysis buffer (phosphate buffer $50 \mathrm{mM}, \mathrm{NaCl} 150 \mathrm{mM}, \mathrm{MgCl}_{2} 2.5 \mathrm{mM}$, protease inhibitor, DNase, $\mathrm{pH}$ 7.4) and sonicated ( 5 min at $20 \%$ of amplitude, $3 \mathrm{sec}$ on, $1 \mathrm{sec}$ off). The lysate was incubated for $2 \mathrm{~h}$ at $4{ }^{\circ} \mathrm{C}$ to allow DNA digestion by DNase. Cellular fragments were removed by centrifugation $\left(9200 \times \mathrm{g}\right.$ for $1 \mathrm{~h}$ at $\left.4^{\circ} \mathrm{C}\right)$. The supernatant was incubated overnight at $4^{\circ} \mathrm{C}$ under gentle agitation with Ni-NTA agarose beads in phosphate buffered saline (PBS) (sodium phosphate $50 \mathrm{mM}, \mathrm{NaCl} 150 \mathrm{mM}, \mathrm{pH}$ 7.4) complemented with $10 \mathrm{mM}$ imidazole. Beads were washed with 20 volumes of PBS containing $20 \mathrm{mM}$ imidazole, and with 5 volumes of PBS complemented with $40 \mathrm{mM}$ imidazole. His-tagged proteins were eluted with 5 volumes of PBS complemented with $0.5 \mathrm{M}$ imidazole. The buffer was exchanged to PBS (50 mM phosphate, $150 \mathrm{mM} \mathrm{NaCl}, \mathrm{pH} 7.4$ ) using PD-10 desalting columns.

Physico-chemical Measurements Thermodynamic dissociation constants were determined with a Spark 10M plate reader (Tecan) and fit in Prism 6 to a one-site specific 
binding model. Steady state UV-Vis absorption spectra were recorded using a Cary 300 UV-Vis spectrometer (Agilent Technologies), equipped with a Versa20 Peltier-based temperature-controlled cuvette chamber (Quantum Northwest) and fluorescence data were recorded using a LPS 220 spectrofluorometer (PTI, Monmouth Junction, NJ), equipped with a TLC50TM Legacy/PTI Peltier-based temperature-controlled cuvette chamber (Quantum Northwest). The quantum yields of fluorescence $\left(\Phi_{F}\right)$ for the variant:fluorogen complexes were determined using different concentrations of fluorogen and a constant concentration of FAST variant $(40 \mu \mathrm{M})$, such that the complex formed was always $\sim 100 \%$. Complex absorbance was kept below 0.05 and plotted as a function of the integrated emission intensity according to the equation:

$$
\Phi_{F}^{S}=\Phi_{F}^{R} \cdot \frac{I_{S}}{I_{R}} \cdot \frac{A b s_{R}}{A b s_{S}} \cdot\left(\frac{n^{S}}{n^{R}}\right)^{2}
$$

where $S$ and $R$ are the sample and reference, respectively, $I$ is the integrated intensity of fluorescence emission, Abs is the absorbance at the excitation wavelength and $n$ is the refractive index of each solution. FAST:HMBR was used as a reference.

Mammalian cell culture HEK 293T cells were cultured in Dulbecco's Modified Eagle Medium (DMEM) supplemented with phenol red, Glutamax I, and 10\% (vol/vol) fetal calf serum (FCS), at $37^{\circ} \mathrm{C}$ in a $5 \% \mathrm{CO}_{2}$ atmosphere. For imaging, cells were seeded in $\mu$ Dish IBIDI (Biovalley) coated with poly-L-lysine. Cells were transiently transfected using Genejuice (Merck) according to the manufacturer's protocol for 24 hours prior to imaging.

Fluorescence microscopy Confocal micrographs were acquired on a Zeiss LSM 710 Laser Scanning Microscope equipped with a Plan Apochromat 63×/1.4 NA oil immersion objective. Cells were imaged in DMEM supplemented with $20 \mu \mathrm{M}$ HBR-3OM for quantification. Images were collected as a series of z-stacks by defining the first and last image whereby positive intensity is detected so as to capture all in and out of focus light. The number of images varied on the thickness of the cells but the imaging interval between stacks was kept constant for all stacks. Stacks were analyzed by generating a sum intensity projection across the stack and the integrated density was measured for each cell for about 80-90 cells per sample. The experiment was repeated four times, with 
a total number of cells analyzed of $373,324,374$, and 329 for FAST, iFAST, td-iFAST, and td-FAST, respectively.

Photobleaching measurements for HMBR, HBR-3,5DM, and HBR-3OM were carried out at $10 \mu \mathrm{M}$ fluorogen at $488 \mathrm{~nm}$ excitation ( $4.6 \mathrm{~kW} / \mathrm{cm}^{2}, 1.27 \mu \mathrm{sec}$ pixel dwell); EGFP was used as a control. HBR-3,5DOM and mCherry were excited at $541 \mathrm{~nm}\left(2.7 \mathrm{~kW} / \mathrm{cm}^{2}\right.$, $1.27 \mu$ sec pixel dwell). Samples were imaged continuously for 500 images.

Western Blots Samples used for microscopy were washed twice with Dulbecco's Phosphate-Buffered Saline (DPBS) and lysed in 100 uL CellLytic (Sigma). The samples were then centrifuged for 5 minutes at $6,000 \times g$ at $4{ }^{\circ} \mathrm{C}$ and the supernatant was used for further analysis. Total protein concentration was determined by BCA assay (Sigma) in parallel with purified protein samples. Samples were fixed to have the same amount of total protein and separated by SDS-PAGE (4-12\% Bis-Tris NuPAGE). Proteins were transferred by semi-dry blotting to a nitrocellulose membrane (Amersham Protran 0.2 $\mu \mathrm{m})$. Membranes were imaged with Ponceau-S (Sigma) to ensure homogenous transfer and loading and then incubated with blocking buffer (Tris Buffer Saline with $0.1 \%$ Tween20 [TBS-T] $+5 \%$ dry milk) for $1 \mathrm{hr}$. Membranes were incubated overnight at $4{ }^{\circ} \mathrm{C}$ with antimyc (Cell Signaling Technology) at 1:2000 in blocking buffer and then washed with TBST for 1 hour. The membranes were then stained with diluted (1:2500) secondary antibody, anti-rabbit (Cell Signaling Technology), in blocking buffer for one hour. Membranes were then washed for 1 hour in TBS-T. Antibody was visualized with BioRad ClarityMax reagent after 5 min incubation using a BioRad ChemiDoc MP. Blots were analyzed in ImageJ using the gel analyzer tool without background correction. Standard curves using purified FAST-myc and td-FAST-myc were used to interpolate the quantity of protein in the sample. 


\section{References}

(1) Plamont, M.-A., Billon-Denis, E., Maurin, S., Gauron, C., Pimenta, F. M., Specht, C. G., Shi, J., Querard, J., Pan, B., Rossignol, J., Morellet, N., Volovitch, M., Lescop, E., Chen, Y., Triller, A., Vriz, S., Le Saux, T., Jullien, L., and Gautier, A. (2016) Small fluorescence-activating and absorption-shifting tag for tunable protein imaging in vivo. Proc. Natl. Acad. Sci. U. S. A 113, 497-502.

(2) Li, C., Plamont, M.-A., Sladitschek, H. L., Rodrigues, V., Aujard, I., Neveu, P., Le Saux, T., Jullien, L., and Gautier, A. (2017) Dynamic multicolor protein labeling in living cells. Chem. Sci. 8, 5598-5605. 